\title{
RELATIVE SIZE OF THE SHILOV BOUNDARY OF A FUNCTION ALGEBRA
}

\author{
WILLIAM R. ZAME
}

\begin{abstract}
A notion of size for subsets of the spectrum of a function algebra is described, relative to which each open subset of the Shilov boundary has the same size as the spectrum.
\end{abstract}

Let $A$ be a function algebra; denote its spectrum (space of nonzero, continuous, complex-valued homomorphisms, with the weak*-topology) by $\Sigma_{A}$, and its Shilov boundary by $\Gamma_{A}$. In a topological sense, $\Gamma_{A}$ may be much smaller than $\sum_{A}$; for instance it is easy to construct function algebras for which $\Gamma_{A}$ is zero-dimensional, while the dimension of $\Sigma_{A}$ is as large as we please (see Stout [8, p. 372]). In this note we describe another natural notion of size, an analytic notion derived from the theory of several complex variables, relative to which $\Gamma_{A}$ and $\Sigma_{A}$ have precisely the same size. This generalizes and simplifies some of the recent results of Huckleberry and Stoll [3]. The proof uses techniques similar to the function algebra techniques in [3].

We will make use of the $A$ sholomorphic functions introduced by Rickart [4], [5], [6], which may be defined by transfinite induction as follows: Let $A_{0}=A$, and assume that the class $A_{v}$ has been defined for all ordinal numbers $\nu<\mu$. A function $f$ defined on a subset $E$ of $\Sigma_{A}$ will belong to the class $A_{\mu}$ if for each $x \in E$ there is a compact neighborhood $K$ of $x$, such that $f \mid(K \cap E)$ is the uniform limit of functions which are defined on $K \cap E$ and belong to classes $A_{\nu}$ for $v<\mu$. We say that a function $h$ defined on a subset $E$ of $\Sigma_{A}$ is $A$-holomorphic if it is an element of the class $A_{\mu}$ for some ordinal number $\mu$ and denote the collection of such functions by $\mathscr{H}_{A}(E)$; we refer to Rickart [6] for general information about $A$-holomorphic functions (and to Stout [8] for general facts concerning function algebras). The fundamental result we use is the following

THEOREM. Let $A$ be a function algebra with spectrum $\Sigma_{A}$ and Shilov boundary $\Gamma_{A}$. Then $\mathscr{H}_{A}\left(\Sigma_{A}\right)$ is a function algebra with spectrum $\Sigma_{A}$ and Shilov boundary $\Gamma_{A}$.

Received by the editors May 1, 1973.

AMS (MOS) subject classifications (1970). Primary 46J10, 46J20; Secondary 32E25.

Key words and phrases. Spectrum, Shilov boundary, $A$-holomorphic function, thin sets.

(c) American Mathematical Society 1974 
If $\Omega$ is an open subset of $\Sigma_{A}$, then by an $A$-holomorphic variety in $\Omega$ we mean a closed subset $V$ of $\Omega$ such that for each $x \in V$, there are an open set $Q$ containing $x$ and a (not necessarily finite) family $\mathscr{F} \subset \mathscr{H}_{A}(Q)$ for which $V \cap Q=\{y \in Q: f(y)=0$ for all $f \in \mathscr{F}\}$. In analogy with the usual notion of thinness in several complex variables (see e.g. Gunning and Rossi [2, p. 19]) we say that a subset $T$ of $\Sigma_{A}$ is $A$-thin if there is a sequence $V_{1}, V_{2} \cdots$ of $A$-holomorphic varieties defined in open sets $\Omega_{1}, \Omega_{2} \cdots$ respectively, such that $T \subset \bigcup_{i=1}^{\infty} V_{i}$, while $\bigcup_{i=1}^{\infty} V_{i}$ has no interior in $\Sigma_{A}$. Our result is then as follows.

THEOREM. If $A$ is a function algebra with spectrum $\Sigma_{A}$ and Shilov boundary $\Gamma_{A}$, then no relatively open subset of $\Gamma_{A}$ is $A$-thin in $\Sigma_{A}$.

In order to establish the theorem, we will use a slight modification of a result of Glicksberg [1]; the proof is similar to the one in [3].

LEMMA. Let $B$ be a function algebra. If $U$ is a nonempty relatively open subset of $\Gamma_{B}$, then there is an open subset $U^{\prime}$ of $\Sigma_{B}$ for which

$$
\varnothing \neq U^{\prime} \cap \Gamma_{B} \subset U
$$

and $f \mid U^{\prime} \equiv 0$ for each $f \in B$ having the property that $f \mid U \equiv 0$.

Proof. Since $U$ is relatively open, it contains a strong boundary point, $x_{0}$ say. We can find a function $g \in B$ such that $g\left(x_{0}\right)=\|g\|=1$, while $|g(y)|<1$ for $y \in\left(\Gamma_{B}-U\right)$. Set $\alpha=\max \left\{|g(y)|: y \in\left(\Gamma_{B}-U\right)\right\}, U^{\prime}=\left\{x \in \Sigma_{B}\right.$ : $|g(x)|>\alpha\}$. Then $U^{\prime}$ is certainly open, $x_{0} \in\left(U^{\prime} \cap \Gamma_{B}\right)$ and $\left(U^{\prime} \cap \Gamma_{B}\right) \subset U$.

Now let $f \in B$ such that $f \mid U \equiv 0$. If $\alpha \neq 0$, then for every positive integer $n$, set $h_{n}=\alpha^{-n} g^{n} f$. It is easy to see that $\left|h_{n}(x)\right| \leqq\left|f_{n}(x)\right|$ for each $x \in \Gamma_{B}$, so that $\left\|h_{n}\right\| \leqq\|f\|$. If $x \in U^{\prime}$ then $|g(x)|>\alpha$ and

$$
|f(x)|=\alpha^{n}\left|h_{n}(x)\right||g(x)|^{-n} \rightarrow 0
$$

as $n \rightarrow \infty$; i.e. $f(x)=0$. Finally, if $\alpha=0$, then $f g(x)=0$ for each $x \in \Gamma_{B}$, so that $f g \equiv 0$, and again $f \mid U^{\prime} \equiv 0$, which completes the proof.

ProOF OF THE THEOREM. Let $V_{1}, V_{2} \cdots$ be a sequence of $A$-holomorphic varieties in open sets $\Omega_{1}, \Omega_{2} \cdots$ such that $\bigcup_{i=1}^{\infty} V_{i}$ contains a relatively open subset of $\Gamma_{A}$; we show that $\bigcup_{i=1}^{\infty} V_{i}$ has interior in $\Sigma_{A}$. Observe first that the Baire category theorem implies that one of these $A$-holomorphic varieties, say $V_{1}$, contains a nonempty relatively open set $U_{1} \subset \Gamma_{A}$. Let $x_{1} \in U_{1}$; there are an open set $Q$ containing $x$ and a family $\mathscr{F} \subset \mathscr{H}_{A}(Q)$ such that $Q \cap \Gamma_{A} \subset U_{1}$ and $V_{1} \cap Q=\{y \in Q: f(y)=0$ for all $f \in \mathscr{F}\}$. We can choose a compact, $A$-convex neighborhood $K$ of $x_{1}$ with $K \subset Q$. Let $A^{\prime}$ be the uniform completion of $A \mid K$; then $A^{\prime}$ is a function algebra, $\Sigma_{A^{\prime}}=K$, and Rossi's local maximum modulus principle [7] implies that $\Gamma_{A^{\prime}} \subset$ $\left(K \cap \Gamma_{A}\right) \cup \partial K$, where $\partial K$ denotes the topological boundary of $K$ in $\Sigma_{A}$. 
Set $B=\mathscr{H}_{A^{\prime}}(K)$; then $B$ is a function algebra, $\Sigma_{B}=K, \Gamma_{B}=\Gamma_{A^{\prime}} \subset$ $\left(K \cap \Gamma_{A}\right) \cup \partial K$, and $(h \mid K) \in B$ for each $h \in \mathscr{H}_{A}(Q)$. Since $K$ is a neighborhood of $x_{1}$, there is a relatively open subset $U$ of $\Gamma_{B}$ such that $x_{1} \in U$, $U \subset Q \cap \Gamma_{A} \subset U_{1}$. The Lemma now provides an open subset $U^{\prime}$ of $K$ which contains $x_{1}$ such that $h \mid U^{\prime} \equiv 0$ for each $h \in(\mathscr{F} \mid K)$; thus $V_{1}$ contains an open subset of $\Sigma_{A}$, as desired.

To derive the Huckleberry-Stoll result from the above, let $X$ be a compact polynomially convex subset of $C^{N}$ and let $P(X)$ be the uniform completion on $X$ of the polynomials.

Corollary (HUCKLeberry-Stoll [3]). If $V_{1}, V_{2} \cdots$ is a sequence of varieties in open subsets of $C^{N}$, and $\bigcup_{j=1}^{\infty} V_{j}$ contains a relatively open subset of $\Gamma_{P(X)}$, then $\bigcup_{j=1}^{\infty} V_{j}$ contains a relatively open subset of $X$.

Proof. Observe that if $\Omega$ is open in $C^{N}$ and $f$ is holomorphic on $\Omega$, then $f \mid(\Omega \cap X)$ is $P(X)$-holomorphic; thus each of $V_{1} \cap X, V_{2} \cap X, \cdots$ is a $P(X)$-holomorphic variety. The Corollary now follows immediately.

\section{REFERENCES}

1. I. Glicksberg, Maximal algebras and a theorem of Radó, Pacific J. Math. 14 (1964), 919-941. MR 29 \#6337.

2. R. C. Gunning and H. Rossi, Analytic functions of several complex variables, Prentice-Hall Series in Modern Analysis, Prentice-Hall, Englewood Cliffs, N.J., 1965. MR 31 \#4927.

3. A. Huckleberry and W. Stoll, On the thickness of the Shilov boundary, Math. Ann. (to appear).

4. C. E. Rickart, Analytic phenomena in general function algebras, Pacific J. Math. 18 (1966), 361-377. MR 336438.

5. - The maximal ideal space of functions locally approximable in a function algebra, Proc. Amer. Math. Soc. 17 (1966), 1320-1326. MR 34 \#1876.

6. - Holomorphic convexity for general function algebras, Canad. J. Math. 20 (1968), 272-290. MR 37 \#3362.

7. H. Rossi, The local maximum modulus principle, Ann. of Math. (2) 72 (1960), 1-11. MR 22 \#8317.

8. E. L. Stout, The theory of uniform algebras, Bogden and Quigley, 1971.

Department of Mathematics, SUNY at Buffalo, Amherst, New York 14226 